\title{
Prospective study of lung function and abdominal aortic aneurysm risk: The Atherosclerosis Risk in Communities study
}

\author{
Yasuhiko Kubota a, *, Aaron R. Folsom a , Kunihiro Matsushita ${ }^{\text {b }}$, David Couper ${ }^{\text {, }}$ \\ Weihong Tang ${ }^{a}$ \\ a Division of Epidemiology and Community Health, School of Public Health, University of Minnesota, Minneapolis, MN, USA \\ ${ }^{\mathrm{b}}$ Department of Epidemiology and the Welch Center for Prevention, Epidemiology and Clinical Research, Johns Hopkins Bloomberg School of Public Health, \\ Baltimore, MD, USA \\ ${ }^{c}$ Department of Biostatistics, Gillings School of Global Public Health, University of North Carolina at Chapel Hill, NC, USA
}

\section{A R T I C L E I N F O}

\section{Article history:}

Received 12 September 2017

Received in revised form

6 October 2017

Accepted 10 October 2017

Available online 13 October 2017

\section{Keywords:}

Lung function

Abdominal aortic aneurysm

COPD

Epidemiology

\begin{abstract}
A B S T R A C T
Background and aims: No prospective study has investigated whether individuals with respiratory impairments, including chronic obstructive pulmonary disease (COPD) and restrictive lung disease (RLD), are at increased risk of abdominal aortic aneurysm (AAA). We aimed to prospectively investigate whether those respiratory impairments are associated with increased AAA risk.

Methods: In 1987-1989, the Atherosclerosis Risk in Communities (ARIC) study followed 14,269 participants aged 45-64 years, without a history of AAA surgery, through 2011. Participants were classified into four groups, "COPD" [forced expiratory volume in $1 \mathrm{~s}$ (FEV1)/forced vital capacity (FVC) <lower limit of normal (LLN)], "RLD" (FEV1/FVC $\geq$ LLN and FVC < LLN), "respiratory symptoms with normal spirometry" (without RLD or COPD), and "normal" (without respiratory symptoms, RLD or COPD, reference group).

Results: During the 284,969 person-years of follow-up, 534 incident AAA events were documented. In an age, sex, and race-adjusted proportional hazards model, individuals with respiratory impairments had a significantly higher risk of AAA than the normal reference group. After adjustment for AAA risk factors, including smoking status and pack-years of smoking, AAA risk was no longer significant in the respiratory symptoms with normal spirometry group [HR $(95 \% \mathrm{CI}), 1.25(0.98-1.60)$ ], but was still increased in the other two groups [RLD: 1.45 (1.04-2.02) and COPD: 1.66 (1.34-2.05)]. Moreover, continuous measures of FEV1/FVC, FEV1 and FVC were associated inversely with risk of AAA.

Conclusions: In the prospective population-based cohort study, obstructive and restrictive spirometric patterns were associated with increased risk of AAA independent of smoking, suggesting that COPD and RLD may increase the risk of AAA.
\end{abstract}

(c) 2017 Elsevier B.V. All rights reserved.

\section{Introduction}

Abdominal aortic aneurysm (AAA) is a common disease in Western populations, especially in elderly people [1]. Once rupture occurs, mortality rates can be as high as $65-85 \%$ [2]. So far, there is no established treatment for AAA other than surgery. Since AAA is usually asymptomatic and AAA growth is discontinuous, with periods of growth alternating with periods of stability, it is often

\footnotetext{
* Corresponding author. Division of Epidemiology and Community Health, School of Public Health, University of Minnesota, 1300 South 2nd Street, Minneapolis, MN 55454, USA.

E-mail address: kubot007@umn.edu (Y. Kubota).
}

difficult to estimate the prognosis of AAA or offer interventions [3]. Thus, it is important to identify populations with high risk of AAA.

Some research has suggested that chronic obstructive pulmonary disease (COPD) is associated with increased risks of AAA and its rupture [4-9]. A previous clinical cross-sectional study showed that this association was independent of smoking, and mediated by inflammation and hemostasis [9]. A previous population-based study showed that circulating inflammatory and hemostatic markers are prospectively associated with AAA events [10], and thus, inflammatory lung diseases, like COPD or restrictive lung diseases (RLD), as sources of circulating inflammatory products, may contribute to AAA. However, to the best of our knowledge, no prospective epidemiological study has examined the association 
between those respiratory impairments and AAA.

Therefore, the objective of this study was to prospectively investigate whether respiratory impairments including COPD and RLD, assessed by spirometry and respiratory symptoms, are associated with increased incidence of AAA in a population-based study in the U.S., the Atherosclerosis Risk in Communities (ARIC) study.

\section{Materials and methods}

\subsection{Study population}

The ARIC study is an ongoing population-based prospective investigation aimed to identify risk factors for cardiovascular diseases [11]. In 1987-1989, the ARIC study recruited and examined 15,792 mostly white or African American men and women aged 45-64 years, within 4 U.S. communities (Washington County, MD; Forsyth County, NC; Jackson, MS (African Americans only); and suburbs of Minneapolis, MN).

The ARIC study was approved by the institutional review boards of the collaborating universities, and written informed consent was obtained from all participants.

\subsection{Main exposure: lung function and respiratory symptoms}

ARIC conducted spirometry at visit 1 baseline using a watersealed Collins Survey II volume displacement spirometer (Collins Survey II; Collins Medical; Braintree, MA) and Pulmo-Screen II software (Pulmo-Screen; PDS Healthcare Products; Louisville, CO) [12]. Using the Hankinson 1999 equations, forced expiratory volume in $1 \mathrm{~s}\left(\mathrm{FEV}_{1}\right)$ as a percentage of predicted value $\left(\mathrm{FEV}_{1} \%\right.$ predicted), forced vital capacity (FVC) as a percentage of predicted value (FVC \% predicted), and the lower limit of normal (LLN) were calculated [13]. ARIC did not evaluate bronchodilator (beta-agonist) response at visit 1 .

Respiratory symptoms were assessed using a standardized selfadministered questionnaire [14]. Participants were considered as having respiratory symptoms when they answered positively to any of the following questions [15]: "Do you usually have a cough?"; "Do you usually bring up phlegm from your chest?"; "Does your chest ever sound wheezy or whistling apart from colds?"; "Do you have to walk slower than people of your age on the level because of breathlessness?"; and "Are you too breathless to leave the house or breathless on dressing or undressing?".

Participants were classified into 4 categories as follows: "Normal" was defined as those with $\mathrm{FEV}_{1} / \mathrm{FVC} \geq$ LLN, FVC $\geq$ LLN and no respiratory symptoms, "respiratory symptoms with normal spirometry" as those with respiratory symptoms but $\mathrm{FEV}_{1} /$ FVC $\geq$ LLN and FVC $\geq$ LLN, "RLD pattern" as FEV $1 / F V C \geq$ LLN and FVC < LLN, and "COPD pattern" as FEV $_{1} /$ FVC < LLN [15].

\subsection{Potential confounding factors}

Potential confounding factors included age, sex, race (white or African American), height (cm), smoking status (current, former or never), pack-years of smoking, hypertension (systolic blood pressure $\geq 140 \mathrm{mmHg}$, diastolic blood pressure $\geq 90 \mathrm{mmHg}$ or hypertension medication use) [16], diabetes mellitus (a fasting blood glucose $\geq 126 \mathrm{mg} / \mathrm{dl}$, non-fasting blood glucose $\geq 200 \mathrm{mg} / \mathrm{dl}$, a selfreported physician diagnosis of diabetes, or use of antidiabetic medication in the past 2 weeks) [17], HDL cholesterol (mmol/L), LDL cholesterol ( $\mathrm{mmol} / \mathrm{L}$ ), and history of peripheral artery disease. Since our previous study showed that obesity was not associated with AAA risk [18], weight and body mass index were not considered as potential confounders, although they were presented in baseline characteristics.

\subsection{Ascertainment of abdominal aortic aneurysm}

Incident clinical AAAs were identified by the following several strategies [10,18]: ARIC interviewers asked with annual telephone calls about any interim hospitalizations and identified deaths. ARIC also surveyed local hospitals to identify additional hospitalizations or deaths. In addition, ARIC linked participant identifiers with feefor-service Medicare data from the Centers for Medicare and Medicaid Services for 1991-2011, to find additional hospital or outpatient events for those over 65 years. Incident clinical AAAs were defined as those with a hospital discharge diagnosis from any source, or two Medicare outpatient claims that occurred at least one week apart, with ICD-9-CM codes of 441.3 or 441.4 , or procedure codes of 38.44 or 39.71 , or death codes of ICD-9441.3 or 441.4 or ICD-10 code I71.3 or I71.4. AAAs based on procedure codes were required to be verified by diagnosis codes. Some of these clinical diagnoses would include asymptomatic AAAs medically documented. Thoracic, thoracoabdominal, or unspecified aortic aneurysms were treated as non-events.

\subsection{Statistical analysis}

SAS version 9.4 software (SAS Institute Inc., Cary, NC) was used for statistical analyses. All statistical tests were two-tailed and $p$ values $<0.05$ were considered as significant.

From a total of 15,972 participants, the following participants were excluded; participants who reported prior AAA surgery $(\mathrm{n}=11)$ at baseline, had uncertain AAA status during follow-up $(\mathrm{n}=30)$, non-white participants in Washington County or Minneapolis or non-white/black participants in Forsyth County $(\mathrm{n}=48)$ in order to allow multivariable adjustment for race and study site [19], and participants whose data on main exposures ( $n=197)$ or any covariates $(n=1237)$ were missing. Finally, 14,269 participants were included in the present analyses.

Person-years of follow-up were calculated from baseline to the first endpoint: AAA, death (non-AAA related death was censored at the time of death), loss to follow-up, or administrative censoring at December 31, 2011. Hazard ratios (HRs) and their 95\% confidence intervals (CIs) of clinical AAA were calculated using Cox proportional hazard models. The proportional hazards assumption in the Cox regression was tested using risk factor-by-time interactions and was not violated. Since we found no statistical interactions between sex or race and respiratory categories in relation to AAA risk ( $p$ for interaction $>0.05$ ), we pooled the analysis across sex and race. We also found no interaction about age. We constructed cubic spline graphs with 3 knots at 25, 50 and 75 percentiles in order to examine dose-response relationships between continuous measures of FEV1\% predicted, FVC \% predicted or FEV1/FVC and AAA risk.

Several sensitivity analyses were performed: (i) the associations between respiratory impairments and AAA risk were examined after stratifying participants by smoking status, (ii) multiplicative interactions were tested between respiratory impairments and smoking status or pack-years in the overall AAA multivariable model, models were rerun after (iii) excluding AAA cases identified by Medicare data, (iv) excluding early AAA events (within 10 years of baseline) and/or participants who were 60-64 years old to reduce the influence of unidentified AAA at baseline, and (v) excluding participants with diabetes at baseline.

\section{Results}

\subsection{Baseline characteristics according to lung function categories}

Characteristics of this study are shown in Table 1. Individuals 
Table 1

Baseline characteristics of participants according to respiratory impairments, ARIC, 1987-1989 ( $\mathrm{n}=14,269)$.

\begin{tabular}{|c|c|c|c|c|}
\hline & Normal & Respiratory symptoms with normal spirometry & Restrictive lung disease pattern & COPD pattern \\
\hline Participants, n & 8653 & 2280 & 841 & 2495 \\
\hline \multicolumn{5}{|l|}{ AAA risk factors } \\
\hline Age, y & $53.8 \pm 5.7$ & $54.2 \pm 5.7$ & $54.6 \pm 5.6$ & $55.0 \pm 5.8$ \\
\hline Female, \% & 56.3 & 57.8 & 52.1 & 50.3 \\
\hline African American, \% & 26.3 & 27.5 & 19.4 & 21.5 \\
\hline Height, cm & $168.4 \pm 9.3$ & $167.6 \pm 9.1$ & $168.9 \pm 9.3$ & $169.7 \pm 9.5$ \\
\hline Diabetes mellitus, \% & 10.0 & 14.0 & 21.6 & 8.8 \\
\hline Current smoker, \% & 15.3 & 35.3 & 35.1 & 49.2 \\
\hline Pack-years of smoking & $10.8 \pm 17.2$ & $17.8 \pm 22.7$ & $20.7 \pm 23.2$ & $30.2 \pm 26.4$ \\
\hline Hypertension, \% & 32.3 & 38.2 & 45.5 & 30.9 \\
\hline LDL cholesterol, mmol/L & $3.6 \pm 1.0$ & $3.6 \pm 1.0$ & $3.6 \pm 1.1$ & $3.5 \pm 4.8$ \\
\hline HDL cholesterol, mmol/L & $1.4 \pm 0.4$ & $1.3 \pm 0.4$ & $1.2 \pm 0.4$ & $1.4 \pm 0.5$ \\
\hline Peripheral artery disease, \% & 3.1 & 4.4 & 5.8 & 6.5 \\
\hline \multicolumn{5}{|l|}{ Obesity measures } \\
\hline Weight, kg & $78.1 \pm 16.1$ & $80.3 \pm 17.2$ & $86.0 \pm 19.6$ & $75.0 \pm 16.1$ \\
\hline Body mass index, $\mathrm{kg} / \mathrm{m}^{2}$ & $27.6 \pm 5.0$ & $28.7 \pm 5.8$ & $30.2 \pm 6.5$ & $26.0 \pm 4.8$ \\
\hline \multicolumn{5}{|l|}{ Respiratory measures } \\
\hline $\mathrm{FEV}_{1} \%$ predicted & $100.2 \pm 12.1$ & $96.6 \pm 12.1$ & $72.6 \pm 8.1$ & $74.9 \pm 18.2$ \\
\hline FVC \% predicted & $101.6 \pm 11.7$ & $98.9 \pm 11.6$ & $72.8 \pm 7.2$ & $94.0 \pm 17.8$ \\
\hline $\mathrm{FEV}_{1} / \mathrm{FVC}, \%$ & $77.2 \pm 4.7$ & $76.5 \pm 4.8$ & $78.0 \pm 6.2$ & $61.5 \pm 7.7$ \\
\hline Respiratory symptoms, \% & 0 & 100 & 37.9 & 46.7 \\
\hline
\end{tabular}

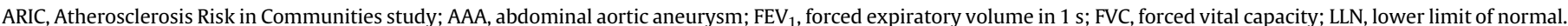

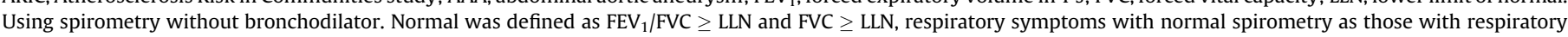
symptoms but $\mathrm{FEV}_{1} / \mathrm{FVC} \geq \mathrm{LLN}$ and FVC $\geq \mathrm{LLN}$, restrictive lung disease pattern as $\mathrm{FEV}_{1} / \mathrm{FVC} \geq \mathrm{LLN}$ and FVC $<$ LLN, and COPD pattern as FEV $1 / F V C<\mathrm{LLN}$.

Values are mean \pm standard deviation for continuous variables and \% for categorical variables.

with respiratory symptoms, the RLD pattern or the COPD pattern tended to be older, current smokers, have more pack-years, and have reported history of peripheral artery disease compared to those in the normal group. Participants with RLD pattern were likely to be heavier, while those with COPD pattern tended to have a lower body weight.

\subsection{Associations of respiratory categories with abdominal aortic aneurysm}

During the 284,969 person-years of follow-up, over a median of 22.5 years, 534 incident, clinical AAA events were documented (Table 2). The age-, sex-, and race-adjusted model (Model 1) showed that individuals with respiratory symptoms and normal spriometry, with the RLD pattern, or with the COPD pattern had increased risk of AAA compared to normal participants. Further adjustment for the other potential confounding factors (Model 2) attenuated those associations, but individuals with the RLD [HR (95\% CI): 1.45 (1.04-2.02)] or COPD [1.66 (1.34-2.05)] pattern still had a significantly elevated risk of AAA.

\subsection{Dose-response relations of continuous spirometry measurements with abdominal aortic aneurysm}

The continuous relations of $\mathrm{FEV}_{1} / \mathrm{FVC}, \mathrm{FEV}_{1} \%$ predicted, and $\mathrm{FVC}$ $\%$ predicted with AAA incidence were assessed using restricted cubic splines to evaluate how the degree of lung restriction or airway obstruction was associated with AAA risk (Fig. 1). Lower levels of all three spriometry measurements were significantly associated with increased risks of AAA (inverse dose-response relations).

\subsection{Sensitivity analyses}

Among current smokers (Table 3), all three respiratory impairments groups (respiratory symptoms and normal spirometry, RLD, and COPD) had significantly higher risks of AAA than the normal group. Similar trends were seen among former and never smokers (Table 3), though the associations were not statistically significant.

$\mathrm{FEV}_{1} / \mathrm{FVC}$ was inversely associated with AAA risk in current and former smokers, and was marginally associated in never smokers $(\mathrm{p}=0.08)$ (Table 4). Inverse associations of $\mathrm{FEV}_{1}$ and FVC with AAA were observed in only current smokers.

There were no significant interactions between smoking status (Tables 3 and 4) or pack-years (data not shown) and the respiratory

Table 2

Hazard ratios and 95\% confidence intervals for incident, clinical abdominal aortic aneurysm according to respiratory impairments, ARIC, 1987-2011 ( $\mathrm{n}=14,269$ ).

\begin{tabular}{|c|c|c|c|c|}
\hline & Normal & Respiratory symptoms with normal spirometry & Restrictive lung disease pattern & COPD pattern \\
\hline No. at risk & 8653 & 2280 & 841 & 2495 \\
\hline Person-years & 179,183 & 44,456 & 15,410 & 45,000 \\
\hline Cases & 219 & 96 & 44 & 175 \\
\hline Model 1 & 1 & $1.86(1.46-2.36)$ & $2.40(1.74-3.32)$ & $3.04(2.49-3.71)$ \\
\hline Model 2 & 1 & $1.25(0.98-1.60)$ & $1.45(1.04-2.02)$ & $1.66(1.34-2.05)$ \\
\hline
\end{tabular}

ARIC, Atherosclerosis Risk in Communities study; FEV $_{1}$, forced expiratory volume in $1 \mathrm{~s}$; FVC, forced vital capacity; LLN, lower limit of normal.

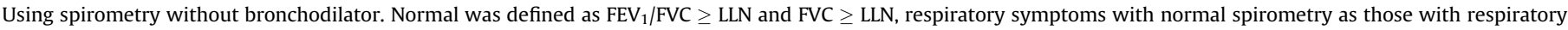
symptoms but $\mathrm{FEV}_{1} / \mathrm{FVC} \geq \mathrm{LLN}$ and FVC $\geq$ LLN, restrictive lung disease pattern as $\mathrm{FEV}_{1} / \mathrm{FVC} \geq$ LLN and FVC $<$ LLN, and COPD pattern as FEV $1 / F V C<$ LLN.

Model 1: adjusted for age, sex, and race/ARIC field center.

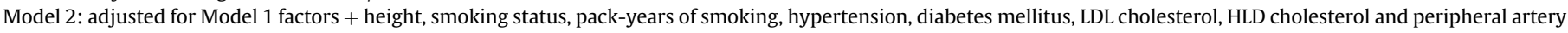
disease. 

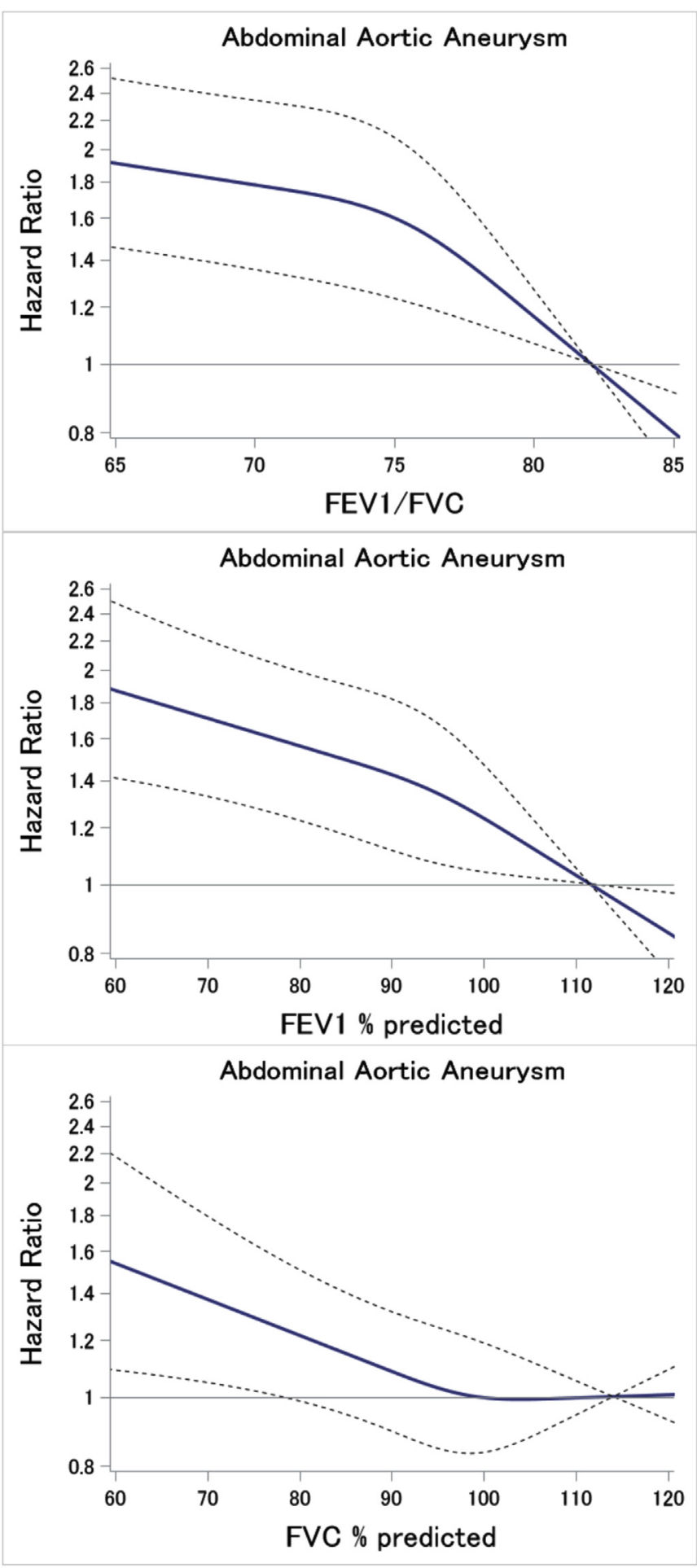

Fig. 1. Multivariably adjusted (Model 2) associations of $\mathrm{FEV}_{1}$ as a percentage of predicted value $\left(\mathrm{FEV}_{1} \%\right.$ predicted), FVC as a percentage of predicted value (FVC \% predicted), or $\mathrm{FEV}_{1} / \mathrm{FVC}$ with $\mathrm{AAA}$

Solid and dashed lines represent hazard ratios and 95\% confidence interval, respectively. The reference value was the median value of the fourth quartile (87.5th percentile).

variables in relation to AAA risk.

Excluding AAA cases identified by Medicare data only $(n=143$, $27 \%$ of total cases), early AAA events, participants who were $60-64$ years old or those with diabetes at baseline produced results similar to the main results (data not shown).

\section{Discussion}

In the prospective population-based ARIC cohort, participants with baseline RLD or COPD patterns had increased risk of incident AAA, independent of other AAA risk factors, including smoking. In addition, lower $\mathrm{FEV}_{1} \%$ predicted and $\mathrm{FEV}_{1} / \mathrm{FVC}$, indices of airway obstruction, and FVC \% predicted, an index of lung restriction, were independently associated with elevated risk of AAA.

AAA is the result of multifactorial processes in the aorta, including inflammation, genetic abnormalities, biomechanical wall stress, apoptosis, and proteolytic degradation of connective tissue, which result in alterations in the structural integrity of the extracellular matrix, leading to AAA formation [20]. Inflammation is considered to be a key component in AAA formation. Several proinflammatory cytokines, including interleukin-6, interleukin$1 \beta$, tumor necrosis factor- $\alpha$, and interferon- $\gamma$, have been identified in AAA walls [21-23]. These cytokines activate lymphocytes and macrophages, and promote matrix metalloproteinase production $[24,25]$. These upregulated matrix metalloproteinases degrade the matrix structural proteins elastin and collagen, leading to AAA. COPD and RLD are inflammatory lung diseases, and they have been associated with elevated markers of systemic inflammation and hemostasis, such as interleukin-6, tumor necrosis factor- $\alpha$ and Ddimer [9,26-30]. Previous studies, mostly cross-sectional, have shown positive associations of plasma inflammatory markers, such as C-reactive protein and interleukin-6 with AAA risk [31]. Inflammatory factors also activate hemostasis, potentially contributing to intraluminal thrombi, which are also important characteristics of AAA [9]. ARIC previously reported that D-dimer was associated with an increased risk of AAA [10]. Thus, increased systemic hemostatic (or thrombogenic) factors from respiratory impairments may also contribute to AAA pathophysiology.

Airway obstruction was particularly strongly associated with increased risk of AAA. Although airway obstruction as seen in COPD affects primarily the right side of the heart (e.g., cor pulmonale in severe COPD), a previous study showed that pulmonary hyperinflation is associated with greater left ventricular mass independent of blood pressure and other traditional CVD risk factors among COPD patients [32], suggesting that COPD may increase the afterload of left ventricle, i.e. aortic pressure (aortic wall stress). In fact, left ventricular hypertrophy has been reported to be associated with a greater diameter of the abdominal aorta, as well as the thoracic aorta [33,34]. This might be another potential mechanism linking airway obstruction with AAA.

Associations between lung function and AAA were qualitatively and statistically (as evidenced by no interaction) similar for current, ever and never smokers, although most associations were not statistically significant for the former and never smoking subgroups, because few AAAs occurred in them. Although this study adjusted for pack-years of smoking, as well as smoking status in the main analysis, the possibility of residual confounding by smoking explaining our findings cannot be completely ruled out.

Compared to the normal group, individuals with respiratory symptoms and normal spirometry, particularly among the current smokers, also had an increased risk of AAA. Respiratory symptoms with preserved lung function may have included borderline COPD or RLD at baseline. In fact, a recent study showed that smokers with respiratory symptoms, but who did not meet spirometric criteria for COPD, had similar adverse health outcomes to those meeting the spirometric definition of COPD [35]. In addition, respiratory symptoms might have reflected underlying inflammatory conditions such as chronic pneumonia. In any case, respiratory 
Table 3

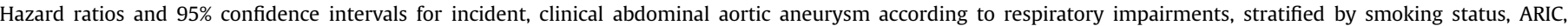
1987-2011 ( $\mathrm{n}=14,269)$.

\begin{tabular}{|c|c|c|c|c|}
\hline & Normal & Respiratory symptoms with normal spirometry & Restrictive lung disease pattern & COPD pattern \\
\hline \multicolumn{5}{|l|}{ Current smokers } \\
\hline No. at risk & 1323 & 804 & 295 & 1228 \\
\hline Person-years & 25,428 & 14,904 & 5279 & 20,077 \\
\hline Cases & 63 & 60 & 27 & 121 \\
\hline Model 2 & 1 & $1.47(1.03-2.11)$ & $1.64(1.04-2.59)$ & $2.05(1.50-2.81)$ \\
\hline \multicolumn{5}{|l|}{ Former smokers } \\
\hline No. at risk & 2952 & 578 & 252 & 761 \\
\hline Person-years & 60,530 & 11,442 & 4410 & 14,274 \\
\hline Cases & 106 & 25 & 14 & 47 \\
\hline Model 2 & 1 & $1.09(0.70-1.69)$ & $1.34(0.76-2.36)$ & $1.37(0.96-1.95)$ \\
\hline \multicolumn{5}{|l|}{ Never smokers } \\
\hline No. at risk & 4378 & 898 & 294 & 506 \\
\hline Person-years & 93,225 & 18,130 & 5721 & 10,649 \\
\hline Cases & 50 & 11 & 3 & 7 \\
\hline Model 2 & 1 & $1.23(0.64-2.37)$ & $1.12(0.35-3.60)$ & $1.20(0.54-2.66)$ \\
\hline$p$ for interaction & 0.48 & & & \\
\hline
\end{tabular}

ARIC, Atherosclerosis Risk in Communities study; FEV $_{1}$, forced expiratory volume in $1 \mathrm{~s}$; FVC, forced vital capacity; LLN, lower limit of normal.

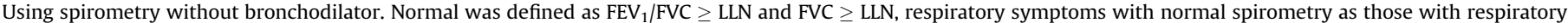
symptoms but $F_{1} V_{1} / F V C \geq L L N$ and FVC $\geq$ LLN, restrictive lung disease pattern as FEV $_{1} / F V C \geq$ LLN and FVC $<$ LLN, and COPD pattern as FEV $1 / F V C<$ LLN.

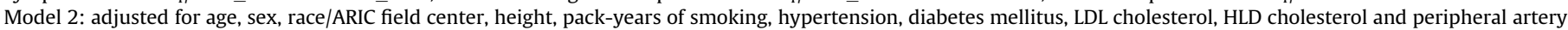
disease.

\section{Table 4}

Hazard ratios and 95\% confidence intervals for incident, clinical abdominal aortic aneurysm according to continuous spirometry measures, stratified by smoking status, ARIC, $1987-2011(\mathrm{n}=14,269)$.

\begin{tabular}{llll}
\hline & $\mathrm{FEV}_{1} / \mathrm{FVC}^{\mathrm{a}}$ & $\mathrm{FEV}_{1} \%$ predicted $^{\mathrm{a}}$ & $\mathrm{FVC}_{\text {o } \text { predicted }^{\mathrm{a}}}$ \\
\hline Current smokers & & & \\
No. at risk & 3650 & $\leftarrow$ & $\leftarrow$ \\
Person-years & 65,687 & $\leftarrow$ & $\leftarrow$ \\
Cases & 271 & $\leftarrow$ & $\leftarrow$ \\
$\quad$ Model 2 & $0.81(0.73-0.90)$ & $0.77(0.69-0.87)$ & $0.86(0.76-0.97)$ \\
Former smokers & & & $\leftarrow$ \\
No. at risk & 4534 & $\leftarrow$ & $\leftarrow$ \\
Person-years & 90,636 & $\leftarrow$ & $\leftarrow$ \\
Cases & 192 & $\leftarrow$ & $0.98(0.83-1.14)$ \\
$\quad$ Model 2 & $0.88(0.77-0.99)$ & $0.89(0.77-1.03)$ & \\
Never smokers & & & $\leftarrow$ \\
No. at risk & 6076 & $\leftarrow$ & $\leftarrow$ \\
Person-years & 127,725 & $\leftarrow$ & $1.02(0.79-1.32)$ \\
Cases & 71 & $\leftarrow$ & 0.15 \\
$\quad$ Model 2 & $0.79(0.60-1.03)$ & $0.90(0.68-1.19)$ & \\
$p$ for interaction & 0.75 & 0.24 & \\
\hline
\end{tabular}

ARIC, Atherosclerosis Risk in Communities study; $\mathrm{FEV}_{1}$, forced expiratory volume in $1 \mathrm{~s}$; FVC, forced vital capacity; LLN, lower limit of normal.

Model 2: adjusted for age, sex, race/ARIC field center, height, pack-years of smoking, hypertension, diabetes mellitus, LDL cholesterol, HLD cholesterol and peripheral artery disease.

a Per 1-SD increment.

symptoms may be a noteworthy risk marker for AAA, particularly among smokers.

Participants with COPD pattern were less likely to have diabetes or hypertension than the normal group, which appears inconsistent with previous reports [36,37]. Although we have no clear reason for this discrepancy, one possible reason might be that those with COPD pattern were less likely to be obese.

Some limitations should be considered. Firstly, the present study assessed lung function and respiratory symptoms only at baseline, and thus, during the follow-up, some participants in the normal group might have developed respiratory impairments. In addition, "COPD pattern" was defined on the basis of spirometry values without the administration of bronchodilator. Although we assumed that the COPD pattern defined in the present study was mainly COPD as in other epidemiological studies [38,39], some participants without lung disease might have been included in the COPD pattern. However, those misclassifications would tend to bias the results toward the null, and thus do not explain the observed associations. Secondly, the assessment of clinical AAA was limited to hospital and death $I C D$ codes $[10,19]$. Although there is no direct evidence of the validity of ICD codes for AAA, the AAA codes seem quite specific, but also insensitive for capturing AAA events. Thus, the approach used in this study might have led to an underestimation of AAA events. Thirdly, the few participants were excluded who had AAA repair before baseline without baseline ultrasound screening. Thus, some participants with prevalent AAA might have been included in the present study. However, given the fact that ARIC participants were $45-65$ years old at baseline, and sensitivity analyses produced results similar to the main results, the number of prevalent AAAs mistakenly included should have been low. Finally, we excluded a relatively large number of participants from the analyses. Although we found excluded participants had characteristics similar to analyzed participants, we cannot deny the possibility that this might have led to bias.

In conclusion, in this prospective biracial cohort, obstructive and restrictive spirometric patterns were associated with increased risk of AAA independent of smoking, suggesting that COPD and RLD may increase the risk of AAA.

\section{Conflict of interest}

The authors declared they do not have anything to disclose regarding conflict of interest with respect to this manuscript.

\section{Financial support}

The Nippon Foundation provided grants to support Dr. Kubota's fellowship at School of Public Health, University of Minnesota. The National Heart, Lung, and Blood Institute (NHLBI) supported ARIC via contracts HHSN268201100005C, HHSN268201100006C, HHSN268201100007C, HHSN268201100009C, HHSN268201100010C, HHSN268201100008C, HHSN268201100011C, HHSN268201100012C, and R01 HL103695. 


\section{Author contributions}

Authors YK and AF contributed to the conception and design of the work. Authors AF and WT acquired the data, and YK performed the analysis. All authors were involved in the interpretation of data. YK and AF drafted the work, which was critically revised by all other authors. All authors approved the final version of the manuscript.

\section{Acknowledgements}

The authors thank the staff and participants of the ARIC study for their important contributions.

\section{References}

[1] U.S. Preventive Services Task Force, Screening for abdominal aortic aneurysm: recommendation statement, Ann. Intern Med. 142 (2005) 198-202.

[2] H.A. Ashton, M.J. Buxton, N.E. Day, L.G. Kim, T.M. Marteau, et al., The Multicentre Aneurysm Screening Study (MASS) into the effect of abdominal aortic aneurysm screening on mortality in men: a randomised controlled trial, Lancet 360 (2002) 1531-1539.

[3] R. Limet, N. Sakalihassan, A. Albert, Determination of the expansion rate and incidence of rupture of abdominal aortic aneurysms, J. Vasc. Surg. 14 (1991) $540-548$.

[4] H. Bengtsson, D. Bergqvist, O. Ekberg, L. Janzon, A population based screening of abdominal aortic aneurysms (AAA), Eur. J. Vasc. Surg. 5 (1991) 53-57.

[5] F.C. Smith, G.M. Grimshaw, I.S. Paterson, C.P. Shearman, J.D. Hamer, Ultrasonographic screening for abdominal aortic aneurysm in an urban community, Br. J. Surg. 80 (1993) 1406-1409.

[6] J.L. Cronenwett, T.F. Murphy, G.B. Zelenock, W.M. Whitehouse Jr., S.M. Lindenauer, et al., Actuarial analysis of variables associated with rupture of small abdominal aortic aneurysms, Surgery 98 (1985) 472-483.

[7] F. Sakamaki, H. Oya, N. Nagaya, S. Kyotani, T. Satoh, et al., Higher prevalence of obstructive airway disease in patients with thoracic or abdominal aortic aneurysm, J. Vasc. Surg. 36 (2002) 35-40.

[8] C.A. Meijer, V.B. Kokje, R.B. van Tongeren, J.F. Hamming, J.H. van Bockel, et al. An association between chronic obstructive pulmonary disease and abdominal aortic aneurysm beyond smoking: results from a case-control study, Eur. J. Vasc. Endovasc. Surg. 44 (2012) 153-157, https://doi.org/10.1016/ j.ejvs.2012.05.016.

[9] F.G. Fowkes, C.L. Anandan, A.J. Lee, F.B. Smith, I. Tzoulaki, et al., Reduced lung function in patients with abdominal aortic aneurysm is associated with activation of inflammation and hemostasis, not smoking or cardiovascular disease, J. Vasc. Surg. 43 (2006) 474-480.

[10] A.R. Folsom, L. Yao, A. Alonso, P.L. Lutsey, E. Missov, et al., Circulating biomarkers and abdominal aortic aneurysm incidence: the atherosclerosis risk in communities (ARIC) study, Circulation 132 (2015) 578-585, https://doi.org/ 10.1161/CIRCULATIONAHA.115.016537.

[11] The Atherosclerosis Risk in Communities (ARIC), Study: Design and objectives. The ARIC Investigators, Am. J. Epidemiol. 129 (1989) 687-702.

[12] Atherosclerosis Risk in Communities Study Manual 4: Pulmonary Function, Chapel Hill NNH, Lung, and Blood Institute of the National Institutes of Health, Collaborative Studies Coordinating Center, University of North Carolina, Chapel Hill, NC, 1987.

[13] J.L. Hankinson, J.R. Odencrantz, K.B. Fedan, Spirometric reference values from a sample of the general U.S. population, Am. J. Respir. Crit. Care Med. 159 (1) (1 1999 Jan) 179-187.

[14] B.G. Ferris, Epidemiology standardization project (American thoracic society), Am. Rev. Respir. Dis. 118 (6 Pt 2) (1978) 1-120.

[15] Y. Kubota, S.J. London, M. Cushman, A.M. Chamberlain, W.D. Rosamond, et al., Lung function, respiratory symptoms and venous thromboembolism risk: the Atherosclerosis Risk in Communities Study, J. Thromb. Haemost. 14 (2016) 2394-2401, https://doi.org/10.1111/jth.13525.

[16] Y. Kubota, K.R. Evenson, R.F. MacLehose, N.S. Roetker, C.E. Joshu, et al., Physical activity and lifetime risk of cardiovascular disease and cancer, Med. Sci. Sports Exerc 49 (8) (2017 Aug) 1599-1605, https://doi.org/10.1249/ MSS.0000000000001274.

[17] Y. Kubota, G. Heiss, R.F. MacLehose, N.S. Roetker, A.R. Folsom, Educational attainment and lifetime risk of cardiovascular disease: the atherosclerosis risk in communities study, JAMA Intern. Med. 177 (8) (2017 Aug 1) 1165-1172, https://doi.org/10.1001/jamainternmed.2017.1877.

[18] W. Tang, L. Yao, N.S. Roetker, A. Alonso, P.L. Lutsey, et al., Lifetime risk and risk factors for abdominal aortic aneurysm in a 24-year prospective study: the ARIC study (atherosclerosis risk in communities), Arterioscler. Thromb. Vasc. Biol. 36 (2016) 2468-2477.

[19] Y. Kubota, M. McAdams-DeMarco, A.R. Folsom, Serum uric acid, gout, and venous thromboembolism: the atherosclerosis risk in communities study Thromb. Res. 144 (2016) 144-148, https://doi.org/10.1016 j.thromres.2016.06.020

[20] M.J. Eagleton, Inflammation in abdominal aortic aneurysms: cellular infiltrate and cytokine profiles, Vascular 20 (2012) 278-283, https://doi.org/10.1258/ vasc.2011.201207.

[21] Z. Szekanecz, M.R. Shah, W.H. Pearce, A.E. Koch, Human atherosclerotic abdominal aortic aneurysms produce interleukin (IL)-6 and interferongamma but not IL-2 and IL-4: the possible role for IL-6 and interferongamma in vascular inflammation, Agents Actions 42 (1994) 159-162.

[22] W.H. Pearce, I. Sweis, J.S. Yao, W.J. McCarthy, A.E. Koch, Interleukin-1 beta and tumor necrosis factor-alpha release in normal and diseased human infrarena aortas, J. Vasc. Surg. 16 (1992) 784-789.

[23] K.M. Newman, J. Jean-Claude, H. Li, W.G. Ramey, M.D. Tilson, Cytokines that activate proteolysis are increased in abdominal aortic aneurysms, Circulation 90 (1994) II224-II227.

[24] N. Malik, B.W. Greenfield, A.F. Wahl, P.A. Kiener, Activation of human monocytes through CD40 induces matrix metalloproteinases, J. Immunol. 156 (1996) 3952-3960.

[25] S. Lacraz, P. Isler, E. Vey, H.G. Welgus, J.M. Dayer, Direct contact between T lymphocytes and monocytes is a major pathway for induction of metalloproteinase expression, J. Biol. Chem. 269 (1994) 22027-22033.

[26] E.F. Wouters, K.H. Groenewegen, M.A. Dentener, J.H. Vernooy, Systemic inflammation in chronic obstructive pulmonary disease: the role of exacerbations, Proc. Am. Thorac. Soc. 4 (2007) 626-634.

[27] A. Tzouvelekis, G. Kouliatsis, S. Anevlavis, D. Bouros, Serum biomarkers in interstitial lung diseases, Respir. Res, 6 (2005) 78.

[28] S. Gläser, T. Ittermann, B. Koch, H. Völzke, H. Wallaschofski, et al., Airflow limitation, lung volumes and systemic inflammation in a general population, Eur. Respir. J. 39 (2012) 29-37, https://doi.org/10.1183/09031936.00009811.

[29] A.W. Fogarty, S.A. Lewis, T.M. McKeever, G.D. Lowe, L. Clark, et al., The association between blood coagulation activity and lung function: a populationbased study, PLoS One 5 (2010), https://doi.org/10.1371/journal. pone.0015014 e15014.

[30] M.G. Crooks, S.P. Hart, Coagulation and anticoagulation in idiopathic pulmonary fibrosis, Eur. Respir. Rev. 24 (2015) 392-399, https://doi.org/10.1183 16000617.00008414.

[31] J. Golledge, P.S. Tsao, R.L. Dalman, P.E. Norman, Circulating markers of abdominal aortic aneurysm presence and progression, Circulation 118 (2008) 2382-2392, https://doi.org/10.1161/CIRCULATIONAHA.108.802074.

[32] B.M. Smith, S.M. Kawut, D.A. Bluemke, R.C. Basner, A.S. Gomes, et al., Pulmonary hyperinflation and left ventricular mass: the multi-ethnic study of atherosclerosis COPD study, Circulation 127 (2013) 1503-1511, https:// doi.org/10.1161/CIRCULATIONAHA.113.001653, 1511e1-6.

[33] D. Iarussi, A. Caruso, M. Galderisi, F.E. Covino, G. Dialetto, et al., Association of left ventricular hypertrophy and aortic dilation in patients with acute thoracic aortic dissection, Angiology 52 (2001) 447-455.

[34] C. Cuspidi, S. Meani, F. Negri, C. Sala, G. Mancia, Left ventricular hypertrophy and abdominal aorta size in essential hypertension, J. Hypertens. 29 (2011) 1213-1219, https://doi.org/10.1097/HJH.0b013e328345954f.

[35] P.G. Woodruff, R.G. Barr, E. Bleecker, S.A. Christenson, D. Couper, et al., Clinical significance of symptoms in smokers with preserved pulmonary function, N. Engl. J. Med. 374 (2016) 1811-1821, https://doi.org/10.1056/ NEJMoa1505971.

[36] M. Divo, C. Cote, J.P. de Torres, C. Casanova, J.M. Marin, et al., Comorbidities and risk of mortality in patients with chronic obstructive pulmonary disease, Am. J. Respir. Crit. Care Med. 186 (2012) 155-161, https://doi.org/10.1164 rccm.201201-00340C.

[37] M. Cazzola, G. Bettoncelli, E. Sessa, C. Cricelli, G. Biscione, Prevalence of comorbidities in patients with chronic obstructive pulmonary disease, Respiration 80 (2010) 112-119, https://doi.org/10.1159/000281880.

[38] M. Thomsen, T.S. Ingebrigtsen, J.L. Marott, M. Dahl, P. Lange, et al., Inflammatory biomarkers and exacerbations in chronic obstructive pulmonary disease, JAMA 309 (2013) 2353-2361, https://doi.org/10.1001/jama.2013.5732.

[39] D.M. Mannino, K.J. Davis, Lung function decline and outcome in an elderly population, Thorax 61 (2006) 472-477. 